\title{
Mobilização precoce a prática do fisioterapeuta intensivista: intervenções e barreiras
}

\section{Early mobilization intensivist physiotherapy practice: interventions and barriers}

\author{
Francisca Vitória dos Santos Paulo ${ }^{1}$ \\ Márcia Cardinalle Correia Viana² \\ Andrea Stopiglia Guedes Braide 3
}

\author{
Marcus César Silva de Morais 4 (1) \\ Virgínia Maria Bezerra Malveira ${ }^{5}$
}

1'Autora para correspondência. Centro Universitário Christus (Fortaleza). Ceará, Brasil. vitoriapaulofisio@hotmail.com ${ }^{2}$ Hospital Geral Dr. César Cals (Fortaleza). Ceará, Brasil. marciaccorreia@hotmail.com ${ }^{3}$ Escola de Saúde Pública do Ceará (Fortaleza). Ceará, Brasil. andreasgbraide@gmail.com ${ }^{4}$ Hospital Regional Unimed (Fortaleza). Ceará, Brasil. marcus.morais@unimedfortaleza.com.br

${ }^{5}$ Centro Universitário Christus (Fortaleza). Ceará, Brasil.vimaria.fisio@gmail.com

RESUMO | INTRODUÇÃO: Pacientes em Unidade de Terapia Intensiva (UTI) requerem longos períodos de internação, estando submetidos ao imobilismo, que resulta em perda significativa de massa muscular. A mobilização precoce é uma terapêutica realizada no ambiente de UTI e tem como objetivo diminuir o comprometimento funcional decorrente do período de internação. OBJETIVO: Analisar a prática de mobilização precoce realizada pelo fisioterapeuta intensivista, identificar as principais intervenções utilizadas por esses profissionais e descrever as barreiras encontradas que inviabilizam a prática da mobilização precoce, em pacientes internados em unidades de terapia intensiva. METODOLOGIA: Estudo de campo, quantitativo e transversal, realizado entre fevereiro e maio de 2020, com fisioterapeutas intensivistas de três hospitais na cidade de Fortaleza, a saber dois deles são da rede pública (um municipal e outro estadual) e o outro de rede privada. Foram inclusos no estudo fisioterapeutas intensivistas atuantes nos hospitais mencionados e que possuam vínculo com a instituição. Foram excluídos os fisioterapeutas na função de residentes, estagiários e preceptores presentes. Para coleta de dados foi utilizado o formulário eletrônico on-line viabilizada por meio do aplicativo Google Forms. Os dados foram analisados e tabulados através do Software Statistical Package for the Social Sciences (SPSS) versão 20.0. A estatística descritiva, com frequências absolutas e relativas foi utilizada para caracterizar o perfil da amostra estudada. Os testes estatísticos aplicados foram o Qui-Quadrado para determinar diferenças nas respostas dos critérios de segurança e barreiras para implementação da mobilização precoce e o tempo de experiência em unidade de terapia intensiva e o Kruskal-Wallis para comparar as intervenções de mobilização precoce entre grupos de Fisioterapeutas dos três hospitais. RESULTADOS: Participaram da pesquisa 68 fisioterapeutas, a maioria (36,8\%) com tempo de atuação na terapia intensiva de 6 a 10 anos. O gerenciamento da mobilização precoce é realizado em sua maioria apenas pelo fisioterapeuta. Acerca da utilização de escalas funcionais utilizadas em UTI, a Medical Research Council (MRC) foi a mais citada pelos profissionais com $(67,7 \%)$. A estratégia de mobilização mais utilizada foi a sedestação $(91,2 \%)$. O desconforto respiratório foi a situação clínica mais citada para a interrupção da mobilização precoce $(83,8 \%)$. CONCLUSÃO: As intervenções mais frequentes foram a sedestação, uso do cicloergômetro e transferências leito poltrona. As barreiras relacionadas ao paciente foram a instabilidade hemodinâmica, uso de drogas sedativas e analgésicas.

PALAVRAS-CHAVE: Mobilização Precoce. Terapia Intensiva. Fraqueza Muscular. Fisioterapeuta.
ABSTRACT | INTRODUCTION: Patients in the Intensive Care Unit (ICU) require long periods of hospitalization, being subjected to immobilization, which results in significant loss of muscle mass. Early mobilization is a therapy performed in the ICU environment and aims to reduce functional impairment resulting from hospitalization. OBJECTIVE: To analyze the practice of early mobilization performed by the intensive care physiotherapist, identify the main interventions used by these professionals, and describe the barriers found that make early mobilization unfeasible in patients admitted to intensive care units. METHODOLOGY: Field study, quantitative and transversal, carried out between February and May 2020, with intensive care physiotherapists from three hospitals in the city of Fortaleza, namely two of them are from the public network (one municipal and the other state) and the other from the private network. Intensive care physiotherapists working in the mentioned hospitals and who have a link with the institution were included in the study. Physiotherapists in the role of residents, interns, and preceptors present were excluded. For data collection, the online electronic form was made possible through the Google Forms application. The data were analyzed and tabulated using the Statistical Package for the Social Sciences (SPSS) version 20.0. The chi-square statistical tests were to determine differences in the responses of the safety criteria and barriers to the implementation of early mobilization and the length of experience in the intensive care unit and Kruskal-Wallis to compare early mobilization interventions between groups of physiotherapists from the three hospitals. RESULTS: 68 physiotherapists participated in the research, the majority (36.8\%) having worked in intensive care for 6 to 10 years. The management of early mobilization is performed mostly by the physiotherapist. Regarding functional scales used in the ICU, the Medical Research Council (MRC) was the most cited by professionals with (67.7\%). The most used mobilization strategy was sedestation (91.2\%). Respiratory distress was the most frequently cited clinical situation for interrupting early mobilization (83.8\%). CONCLUSION: The most frequent interventions were sedation, the cycle ergometer, and transfers from an armchair bed. The patient-related barriers were hemodynamic instability, use of sedative and analgesic drugs.

KEYWORDS: Early Mobilization. Intensive therapy. Muscle weakness. Physiotherapist. 


\section{Introdução}

Em virtude do período de hospitalização, pacientes críticos, internados em unidades de terapia intensiva (UTI), apresentam diminuição de força muscular. A miopatia, adquirida na UTI, está diretamente associada à diminuição da qualidade de vida e morbimortalidade desse grupo de pacientes. ${ }^{1} \mathrm{~A}$ imobilidade, proveniente da restrição ao leito, acarreta em disfunção severa do sistema musculoesquelético, cardiorrespiratório, gastrointestinal, cutâneo e urinário, resultando na perda de massa muscular e inervação.?

Intervir precocemente é de suma importância para a melhoria das repercussões musculoesqueléticas e respiratórias decorrentes do período de internação. ${ }^{3}$ A mobilização precoce (MP) é uma conduta terapêutica realizada no ambiente da UTI, que tem por objetivo diminuir o comprometimento funcional de pacientes críticos, e quando realizada de maneira segura, pode atenuar estes efeitos deletérios. 4,5

A inserção do fisioterapeuta nas unidades de terapia intensiva (UTI) só ocorreu em 1970 e sua consolidação como integrante da equipe de assistência intensiva tem sido progressiva. ${ }^{-} \mathrm{Na}$ assistência ao paciente crítico, o fisioterapeuta é o responsável pela identificação das desordens cinético-funcionais, bem como determinar o modelo mais correto de intervenção precoce, sua viabilidade, constância, frequência e interrupção.

Contudo, a fim de avaliar a aplicabilidade da MP em UTI, é fundamental o uso de instrumentos validados e experimentados nesse ambiente. ${ }^{2}$ Estes instrumentos possibilitam a mensuração da capacidade do indivíduo em realizar atividades básicas do cotidiano, bem como favorecer ao profissional uma melhor condução da sua conduta terapêutica. ${ }^{10}$

As evidências apontam a conduta de MP como segura e eficaz por possuir efeitos benéficos sobre a capacidade funcional, proporcionando a diminuição do período de internação. ${ }^{11}$ Embora traga benefícios a esses pacientes, a sua realização ainda não é amplamente realizada nas UTI's. 12
Diante disso, o objetivo desse estudo foi analisar a prática de mobilização precoce realizada pelo Fisioterapeuta Intensivista em pacientes internados em unidades de terapia intensiva, bem como identificar as principais intervenções utilizadas por estes profissionais e descrever as barreiras encontradas que inviabilizam a prática da mobilização precoce.

\section{Materiais e métodos}

Trata-se de um estudo de campo, quantitativo e transversal, realizado no período de fevereiro a maio de 2020, com fisioterapeutas intensivistas de três Hospitais na cidade de Fortaleza, a saber dois deles são da rede pública (um municipal e outro estadual), e o outro Hospital participante é da rede privada.

O estudo teve a aprovação do Comitê de Ética em Pesquisa dos Hospitais públicos mencionados com os pareceres $n^{\circ}$ 3.812.319 (CAAE 26223919.4.3001.5047) e 3.748.158 (CAAE 26223919.4.0000.5041). No hospital privado, o estudo foi avaliado pelo Centro de Estudos obtendo-se a carta de anuência para que ocorresse o desenvolvimento do estudo. As normas e diretrizes da Resolução 466/12 do Conselho Nacional de Saúde/Ministério da Saúde foram obedecidas. Participaram do estudo fisioterapeutas intensivistas atuantes nos Hospitais mencionados e que possuam vínculo com a instituição. Foram excluídos os Fisioterapeutas na função de residentes, estagiários e preceptores presentes.

Inicialmente, os participantes foram convidados a participar do estudo pela pesquisadora e os que aceitaram, assinaram o Termo de Consentimento Livre e Esclarecido (TCLE), respondendo ao questionário em uma sala reservada. Em virtude da pandemia da COVID-19, as atividades presenciais foram suspensas. Deu-se continuidade ao estudo utilizado como instrumento para coleta de dados a tecnologia de formulário eletrônico on-line viabilizada por meio do aplicativo Google Forms, pelo qual se viabilizou um formulário individual de fácil e rápido acesso aos participantes. 
Os participantes foram convidados a participar da pesquisa por meio de convites encaminhados aos chefes de serviço e repassados aos profissionais via grupos de WhatsApp. O Termo de Consentimento Livre e Esclarecido (TCLE) foi disponibilizado on-line na página inicial e o participante só teve acesso ao instrumento de coleta de dados caso concordasse em participar da pesquisa. Os participantes tiveram um mês a partir do envio do questionário para respondê-lo de forma anônima e voluntária.

O questionário foi construído por perguntas fechadas versando sobre a prática da MP realizada por fisioterapeutas, no que concerne a utilização de escalas funcionais e recursos para avaliação de força muscular, aos critérios de segurança para iniciar a MP e situações clínicas relevantes para sua interrupção, a identificação dos profissionais envolvidos no seu gerenciamento, as condutas mais utilizadas na prática clínica e barreiras encontradas para realizar os exercícios terapêuticos.

Os dados coletados foram inicialmente tabulados no software Excel 2017 e então transferidos para o software estatístico SPSS (Statistical Package for the Social Sciences) versão 20.0. A estatística descritiva, com frequências absolutas e relativas, foi utilizada para caracterizar o perfil da amostra estudada. Para contemplar o objetivo de compreender a relação entre os critérios de segurança e barreiras para implementação da mobilização precoce e o tempo de experiência em unidade de terapia intensiva foi utilizado o teste não paramétrico Qui-Quadrado, e para comparar as intervenções de mobilização precoce entre grupos de Fisioterapeutas dos três hospitais utilizou-se o teste não paramétrico Kruskal - Wallis Kruskal-Wallis, sendo considerado o $p \leq 0,05$.

\section{Resultados}

Participaram do estudo 68 fisioterapeutas, em três hospitais de Fortaleza, sendo 30 (44,1\%) da rede municipal, $21(30,9 \%)$ da rede estadual e 17 (25\%) da rede privada. O tempo de atuação desses profissionais em unidade de terapia intensiva e a existência de protocolo padronizado de mobilização precoce (MP) em suas respectivas unidades e à utilização de escalas funcionais constam na Tabela 1.

Tabela 1. Tempo de atuação dos profissionais fisioterapeutas, protocolo padronizado de MP e escalas funcionais utilizadas em UTI's em 2020 . $\mathrm{N}=68$

\begin{tabular}{ll}
\hline Tempo de Formação & $\mathbf{N}(\%)$ \\
$<5$ anos & $15(22,1)$ \\
Entre 6 a 10 anos & $26(36,8)$ \\
Entre 11 a 15 anos & $8(11,8)$ \\
Entre 16 a 20 anos & $8(11,8)$ \\
Há mais de 20 anos & $12(17,6)$ \\
Protocolo não padronizado & $35(51,5)$ \\
Escalas Aplicadas & $\mathbf{N}(\%)$ \\
Medica Reserach Council (MRC) & $46(67,7)$ \\
Medida de Independência Funcional (MIF) & $1(1,5)$ \\
Escala Barthel & $1(1,5)$ \\
Core - Set & $1(1,5)$ \\
\hline
\end{tabular}

*68 fisioterapeutas; UTI - Unidade de Terapia Intensiva, MP - Mobilização Precoce, UTI - Unidade de Terapia Intensiva.

Quando questionados sobre a utilização de recursos e/ou escala para avaliação de força muscular periférica nos pacientes hospitalizados, 57 (83,8\%) responderam que utilizam a Medical Research Council (MRC). Na Figura 1 constam as situações clínicas informadas pelos participantes para interrupção da mobilização precoce. 


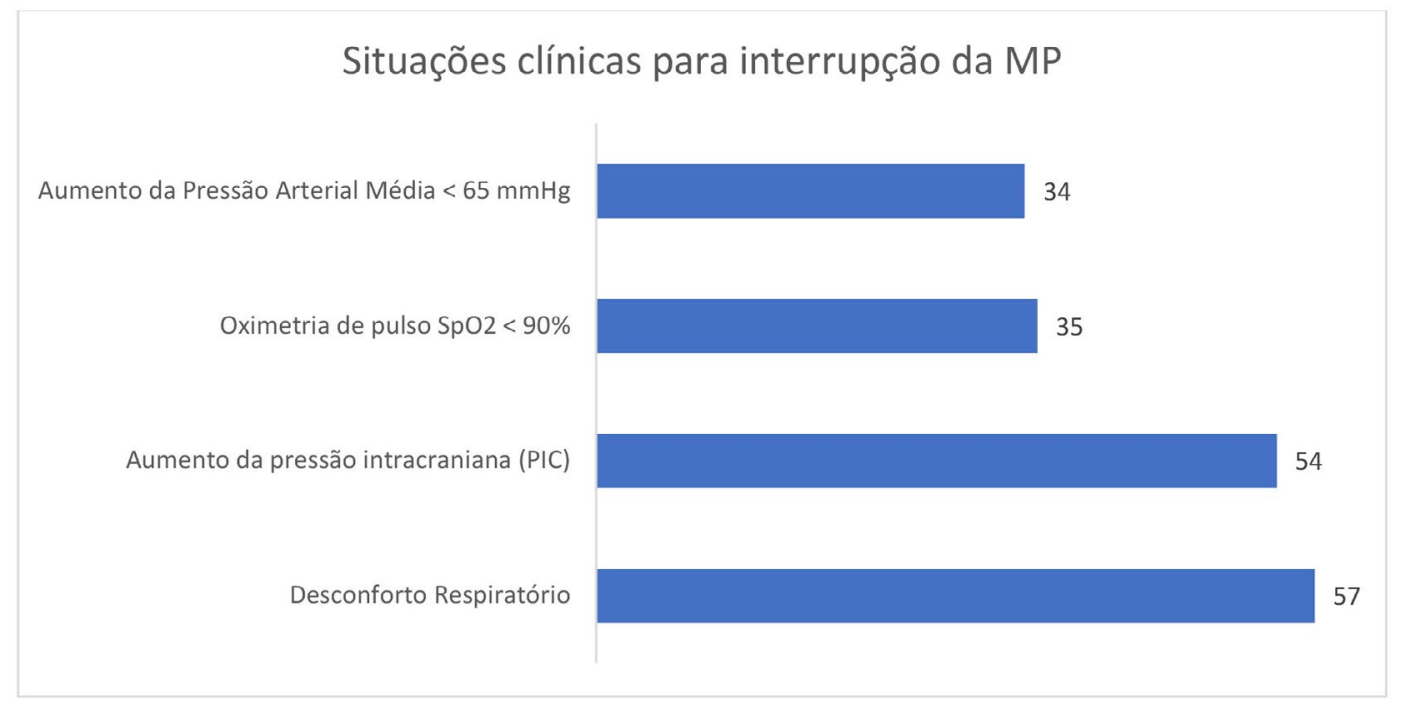

Em relação ao gerenciamento da mobilização precoce (MP), 33 (48,5\%) participantes responderam que apenas o fisioterapeuta era o responsável pela sua implementação, 13 (19,1\%) apontaram os técnicos de enfermagem e $10(14,7 \%)$ responderam que além dos fisioterapeutas, os médicos e enfermeiros também participavam desse gerenciamento.

$\mathrm{Na}$ Tabela 2, encontram-se dados relativos às principais intervenções de MP realizadas por fisioterapeutas intensivistas em hospitais da rede estadual, municipal e privada. Observa-se que as intervenções de deambulação e ortostatismo foram as mais utilizadas para mobilização precoce entre fisioterapeutas intensivistas da rede estadual, municipal e privada, apresentando significância estatística.

Tabela 2. Comparação das intervenções de MP realizadas entre fisioterapeutas intensivistas em da rede hospitalar municipal, estadual e privada -2020. N:68

\begin{tabular}{|c|c|c|c|c|}
\hline Intervenções & $\begin{array}{l}\text { Fisioterapeutas } \\
\text { Rede Estadual (\%) }\end{array}$ & $\begin{array}{l}\text { Fisioterapeutas } \\
\text { Rede Municipal (\%) }\end{array}$ & $\begin{array}{l}\text { Fisioterapeutas } \\
\text { Rede Privada (\%) }\end{array}$ & Valor $(p)$ \\
\hline Sedestação & $21(37,98)$ & $30(33,77)$ & $17(31,5)$ & 0,10 \\
\hline Cicloergômetro & $21(33,71)$ & $30(35,33)$ & $17(31,5)$ & 0,09 \\
\hline Transferência Leito/Poltrona & $21(33,95)$ & $30(34,90)$ & $17(26,50)$ & 0,20 \\
\hline Deambulação & $21(39,29)$ & $30(38,00)$ & $17(29,00)$ & $0,05^{*}$ \\
\hline Ortostatismo & $21(40,40)$ & $30(34,90)$ & $17(26,50)$ & $0,04 *$ \\
\hline Marcha Estacionária & $21(35,79)$ & $30(36,43)$ & $17(29,50)$ & 0,30 \\
\hline
\end{tabular}


Os resultados relacionados aos critérios de segurança e barreiras para implementação da MP estão descritos em correlação com o tempo de atuação dos participantes. A tabela 3 apresenta associação do tempo de atuação dos fisioterapeutas em UTI com os critérios de segurança para realização da MP, não revelando significância estatística entre as variáveis analisadas.

Tabela 3. Tempo de atuação em UTI X critérios de segurança para realização de mobilização precoce. $2020 . \mathrm{N}=68$

\begin{tabular}{|c|c|c|c|c|c|c|}
\hline \multirow{2}{*}{$\begin{array}{l}\text { CRITÉRIOS DE } \\
\text { SEGURANÇA }\end{array}$} & \multicolumn{5}{|c|}{ TEMPO DE ATUAÇÃO EM UTI } & \multirow[b]{2}{*}{ valor $p$} \\
\hline & $<5$ anos & 6-10 anos & $11-15$ anos & 16- 20 anos & $>20$ anos & \\
\hline $\begin{array}{l}\text { Estabilidade } \\
\text { Hemodinâmica }\end{array}$ & $14(21,9 \%)$ & $23(35,9 \%)$ & $8(11,8 \%)$ & $7(10,9 \%)$ & $12(18,8 \%)$ & $p=0,07$ \\
\hline $\mathrm{FR}<24 \mathrm{rpm}$ & $5(23,8 \%)$ & $8(38,1 \%)$ & $3(14,3 \%)$ & $0(0,0 \%)$ & $5(23,8 \%)$ & $p=0,03$ \\
\hline SapO2 $>90 \%$ & $12(31,6 \%)$ & $12(31,6 \%)$ & $5(13,2 \%)$ & $2(5,3 \%)$ & $7(18,4 \%)$ & $p=0,01$ \\
\hline PIC 10- $20 \mathrm{mmHg}$ & $11(24,4 \%)$ & $14(31,1 \%)$ & $6(13,3 \%)$ & $4(8,9 \%)$ & $10(22,2 \%)$ & $p=0,04$ \\
\hline Glasgow $>8$ & $1(6,3 \%)$ & $9(56,3 \%)$ & $2(12,5 \%)$ & $0(0,0 \%)$ & $4(25,0 \%)$ & $p=0,01$ \\
\hline
\end{tabular}

Na tabela 4 estão as principais barreiras para a mobilização precoce correlacionadas ao tempo de atuação em UTI dos profissionais identificadas com maior frequência, observando-se que não houve significância estatística.

Tabela 4. Tempo de atuação em UTI X Barreiras para a realização de mobilização precoce- 2020, N=68

\begin{tabular}{|c|c|c|c|c|c|c|}
\hline \multirow{2}{*}{ BARREIRAS MP } & \multicolumn{5}{|c|}{ TEMPO DE ATUAÇÃO EM UTI: } & \multirow[b]{2}{*}{ valor $p$} \\
\hline & $<5$ anos & $6-10$ anos & $11-15$ anos & 16- 20 anos & $>20$ anos & \\
\hline $\begin{array}{l}\text { Drogas sedativas/ } \\
\text { Analgesia }\end{array}$ & $7(21,2 \%)$ & $9(27,3 \%)$ & $5(15,2 \%)$ & $3(9,1 \%)$ & $9(27,3 \%)$ & $p=0,11$ \\
\hline $\begin{array}{l}\text { Instabilidade } \\
\text { Hemodinâmica }\end{array}$ & $12(21,8 \%)$ & $22(40,0 \%)$ & $6(10,9 \%)$ & $7(12,7 \%)$ & $8(14,5 \%)$ & $p=0,35$ \\
\hline $\begin{array}{l}\text { Indisponibilidade } \\
\text { Profissional }\end{array}$ & $6(25,0 \%)$ & $9(37,5 \%)$ & $4(16,7 \%)$ & $0(0,0 \%)$ & $5(20,8 \%)$ & $p=0,03$ \\
\hline $\begin{array}{l}\text { Falta de protocolos } \\
\text { de Mobilização }\end{array}$ & $3(20,0 \%)$ & $7(46,7 \%)$ & $2(13,3 \%)$ & $1(6,7 \%)$ & $2(13,3 \%)$ & $p=0,09$ \\
\hline Pacientes sob VM & $1(16,7 \%)$ & $2(33,3 \%)$ & $1(16,7 \%)$ & $0(0,0 \%)$ & $2(33,3 \%)$ & $p=0,07$ \\
\hline $\begin{array}{l}\text { Carência de Recursos } \\
\text { Terapêuticos }\end{array}$ & $5(41,7 \%)$ & $3(25,0 \%)$ & $0(0,0 \%)$ & $0(0,0 \%)$ & $4(33,3 \%)$ & $p=0,08$ \\
\hline Uso de DVA & $6(27,3 \%)$ & $8(36,4 \%)$ & $2(9,1 \%)$ & $1(4,5 \%)$ & $5(22,7 \%)$ & $p=0,7$ \\
\hline
\end{tabular}

Quanto à observação da melhora da funcionalidade ao realizar a mobilização precoce, $20(29,4 \%)$ participantes responderam que a observam a através da melhora clínica e evolução médica no prontuário, 27 (39,7\%) através da descrição da evolução de Fisioterapia no prontuário e 55 (80,9\%) através da avaliação por meio de instrumentos ou escalas para avaliação de força muscular. 


\section{Discussão}

O objetivo desse estudo foi analisar a prática de mobilização precoce realizada pelo fisioterapeuta intensivista, identificar as principais intervenções utilizadas por esses profissionais e descrever as barreiras encontradas que inviabilizam a prática da mobilização precoce em pacientes internados em unidades de terapia intensiva. Observa-se que as intervenções de deambulação e ortostatismo têm associação significativa entre os fisioterapeutas intensivistas independente da categoria do hospital. Em relação às barreiras encontradas para realizar essa estratégia, foram relacionadas ao quadro hemodinâmico do paciente e o uso de sedação. Aswegen et al. $\frac{13}{}$ ressaltam que o fisioterapeuta desempenha um papel crucial para o manejo da reabilitação do paciente crítico, sendo de suma importância o esclarecimento das competências necessárias para garantir uma prática clínica segura e eficaz.

No presente estudo, $36,8 \%$ dos participantes informaram tempo de atuação em UTI de 6 a 10 anos. Resultado semelhante foi observado por Nozawa et al. ${ }^{14}$ quando determinaram o perfil profissional do fisioterapeuta em todo o Brasil. Lima et al..$^{15}$ avaliaram o conhecimento dos fisioterapeutas atuantes em UTI adulto sobre as contraindicações à mobilização de pacientes tratados na cidade do Recife.

No que se refere ao gerenciamento da MP, quase metade $(48,5 \%)$ dos participantes apontaram o fisioterapeuta como os únicos responsáveis por essa intervenção. Estudo de Lima et al. ${ }^{15}$ revela que $60 \%$ dos fisioterapeutas são os autores principais desse gerenciamento. França et al. ${ }^{16}$ recomenda que o fisioterapeuta seja o profissional responsável pela implantação e pelo gerenciamento do plano de mobilização, o qual influencia diretamente para o treinamento de futuras atividades funcionais.

De acordo com a resolução $n^{\circ}$ 402/2011 da Especialidade Profissional Fisioterapia e Terapia Intensiva, é de responsabilidade do fisioterapeuta avaliar, prescrever e realizar a reabilitação de pacientes críticos. ${ }^{8} \mathrm{Em}$ nosso estudo, um pequeno percentual $(14,7 \%)$ dos participantes informou que além dos fisioterapeutas, os médicos e enfermeiros também participavam desse gerenciamento. Entretanto, a equipe multidisciplinar deve ser responsável em identificar as indicações e as contraindicações para execução da mobilização precoce para uma avaliação diferenciada e segura..
Em relação à existência de protocolos de MP nas unidades pesquisadas, 51,5\% relatou sua inexistência. No estudo de Koo et al. $\stackrel{17}{ }$, apenas $36 \%$ dos participantes informaram a não existência de protocolos de MP em suas unidades. Sobre esse achado, sabe-se que é de responsabilidade do fisioterapeuta a avaliação cinético funcional e o desenvolvimento de protocolos de MP que visam a otimização da capacidade cardiorrespiratória e a diminuição do declínio funcional do paciente. Ademais, a existência de protocolos orienta os profissionais para implementação dos exercícios terapêuticos trazendo benefícios funcionais aos pacientes. $\frac{18}{.}$ No entanto, a ausência de protocolos de mobilização precoce constitue perdas significativas da funcionalidade do paciente crítico. Sendo assim, a formulação de protocolos clínicos embasados em evidências científicas podem ser referência para a criação de uma ferramenta padronizada que poderá ser benéfica ao desfecho funcional, com impacto na qualidade de vida dessa população. $\underline{\underline{28}}$

Em nossa análise, a maioria $(83,8 \%)$ dos participantes utilizam o MRC como escala de funcionalidade e avaliação força muscular periférica. Vários estudos recomendam a utilização do MRC em pacientes críticos adultos para identificação de fraqueza muscular periférica a fim de minimizar riscos para o declínio funcional. $.16,19$ Sabendo-se que a fraqueza muscular adquirida na unidade de terapia intensiva é considerada uma condição clínica frequente, se apresenta de forma globalizada e simétrica, tanto na musculatura periférica quanto na respiratória, acarretando prejuízos funcionais e aumento do tempo de hospitalização. Baseados nessas premissas torna-se imprescindível a utilização da escala MRC, pois trata-se de uma maneira simples para avaliar força muscular em pacientes críticos. $\frac{16,19}{19}$

A força-tarefa da European Respiratory Society and Europen Societyof Intensive Care Medicine, em 2008, e o Departamento de Fisioterapia da Associação de Medicina Intensiva Brasileira, em 2012, descrevem uma hierarquia de atividades de mobilização na UTI seguindo uma sequência de exercícios que se iniciam a partir da mudança de decúbito, com grau de recomendação Nível $\mathrm{B}$ para mobilização precoce e ortostatismo. $\frac{16,20}{12}$ Nossos resultados revelam que a sedestação $(91,2 \%)$ foi a intervenção para MP mais citada pelos participantes. Segundo Hickmann et al. 1 , quando acompanhada de exercícios ativos, otimiza a aeração pulmonar e minimiza a hipoxemia. 
Outras estratégias de intervenção em nosso estudo foram a utilização do cicloergômetro e transferência do leito para poltrona, também presente no estudo Rocha $\underline{22}$ que investigou os principais protocolos descritos na literatura e técnicas utilizadas. Pires Neto et al. ${ }^{23}$ revelam que um protocolo de cicloergômetro realizado a partir de $72 \mathrm{~h}$ de internação é seguro.

O Departamento de Fisioterapia da Associação de Medicina Intensiva Brasileira publicou em 2012 recomendações mínimas aplicáveis à realidade brasileira, com critérios e mecanismos de segurança na mobilização precoce do doente crítico. ${ }^{16}$ Com relação aos critérios de segurança para a realização da MP em nosso estudo, a estabilidade hemodinâmica foi apontada como principal critério independente do tempo de formação dos profissionais pesquisados. Esse fato pode ser justificado devido a demanda adicional do sistema cardiovascular para manter a pressão arterial e o débito cardíaco em condições que exijam esforço dos pacientes que se encontram restritos ao leito. Em estudo de revisão sistemática conduzido por

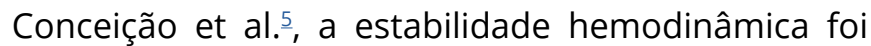
apontada como critério de segurança mais utilizados para iniciar a MP em pacientes críticos sob ventilação mecânica (VM) internados em UTI.

Em um consenso de especialistas multidisciplinares de UTI para buscar um consenso sobre a mobilização segura de pacientes sob ventilação mecânica, as considerações de segurança foram resumidas em quatro categorias: respiratórias, cardiovasculares, neurológicas e clinicas. ${ }^{24}$ Nesse consenso, uma saturação periférica de oxigênio superior a $90 \%$ foi critério para retirada do paciente do leito, visto que é um parâmetro de estabilidade hemodinâmica do paciente, fato observado em nosso estudo como critério de segurança para MP citado pelos profissionais com tempo de atuação na UTI menor que 5 anos.

Dentre as situações clínicas relevantes para a interrupção da mobilização precoce, destacaram-se o desconforto respiratório e aumento da pressão Intracraniana (PIC) em nossos achados. Destaca-se que pacientes com elevação de PIC não são candidatos à conduta de MP. .25 Apesar de ser uma técnica com raros eventos adversos, deve ser realizada com cuidados, evitando complicações cardiorrespiratórias e musculoesqueléticas.
Dubb et al. $\underline{26}$ identificaram 28 barreiras à MP, sendo classificadas em quatro grupos: barreiras estruturais, culturais, relacionadas ao processo de mobilização e as relacionadas ao paciente. Nosso estudo identificou que as barreiras relacionadas ao paciente foram as mais limitantes, porém é importante destacar que existem outras barreiras menos frequentes nesse estudo, como a escassez de recursos terapêuticos para realizar essa prática. A instabilidade hemodinâmica foi a mais citada, em seguida, as drogas sedativas e analgesia. Segundo as Diretrizes Brasileiras de Mobilização Precoce em Unidades de terapia intensiva, a instabilidade hemodinâmica é a maior limitação para realização da mobilização precoce, sendo a hipertensão arterial com PAS $>170 \mathrm{mmHg}$ considerada contraindicação. Embora necessária em algumas situações clínicas, a sedação limita a intervenção do Fisioterapeuta; dessa forma, sua interrupção ou mesmo redução para níveis mínimos é terapêutica, e pode proporcionar melhores desfechos e prognóstico. ${ }^{27}$

A pandemia COVID-19 foi um fator limitante para a realização das entrevistas aos profissionais nos locais de pesquisa, consequentemente, reduziu a amostra analisada. Portanto, se faz necessário estudos mais robustos relacionados a essa abordagem, bem como a implementação de ferramentas que padronizem a conduta de mobilização precoce e facilitem a prática do fisioterapeuta intensivista e o manejo terapêutico ao paciente crítico.

\section{Conclusão}

As intervenções mais frequentes foram a sedestação, uso do cicloergômetro e transferências leito poltrona. As barreiras relacionadas ao paciente foram a instabilidade hemodinâmica, uso de drogas sedativas e analgésicas.

\section{Contribuições dos autores}

Paulo FVS e Viana MCC participaram da criação, coleta de dados, interpretação de resultados e redação do artigo científico. Morais MCS participou da análise estatística e interpretação dos resultados. Braide ASG participou da análise estatística, interpretação dos resultados e redação do artigo científico. Malveira VMB participou da coleta dados e redação do artigo científico. 


\section{Conflitos de interesses}

Nenhum conflito financeiro, legal ou político envolvendo terceiros (governo, empresas e fundações privadas, etc.) foi declarado para nenhum aspecto do trabalho submetido (incluindo, mas não se limitando a subvenções e financiamentos, participação em conselho consultivo, desenho de estudo, preparação de manuscrito, análise estatística, etc.).

\section{Referências}

1. Zhang L, Hu W, Cai Z, Liu J, Wu J, Deng Y, et al. Early mobilization of critically ill patients in the intensive care unit: A systematic review and meta-analysis. PLoS One. 2019;14(10):e0223185. https://doi.org/10.1371/journal.pone.0223185

2. Sarti TC, Vecina MVA, Ferreira PSN. Mobilização precoce em pacientes críticos. J Heal Sci Inst [Internet]. 2016;34(3):177-82. Disponível em: https://pesquisa.bvsalud.org/portal/resource/pt/ biblio-832877

3. Mota CM, Silva VG. A segurança da mobilização precoce em pacientes críticos: uma revisão de literatura. Interfaces Científicas - Saúde e Ambiente. 2012;1(1):83-91. https://doi. org/10.17564/2316-3798.2012v1n1p83-91

4. Lee H, Ko YJ, Suh GY, Yang JH, Park CM, Jeon K, et al. Safety profile and feasibility of early physical therapy and mobility for critically ill patients in the medical intensive care unit: Beginning experiences in Korea. J Crit Care 2015;30(4):673-7. https://doi. org/10.1016/j.jcrc.2015.04.012

5. Conceição TMAD, Gonzáles Al, Figueiredo FCXS, Vieira DSR, Bündchen DC. Safety criteria to start early mobilization in intensive care units. Systematic review. Rev Bras Ter Intensiva. 2017;29(4):509-19. https://doi.org/10.5935/0103-507X.20170076

6. Borges DL, Arruda LA, Rosa TRP, Costa MAG, Baldez TEP, Silva GJP. Influência da atuação fisioterapêutica no processo de ventilação mecânica de pacientes admitidos em UTI no período noturno após cirurgia cardíaca não complicada. Fisioter Pesqui. 2016;23(2):129-35. https://doi.org/10.1590/18092950/14133523022016

7. Harrold ME, Salisbury LG, Webb SA, Allison GT. Early mobilisation in intensive care units in Australia and Scotland: A prospective, observational cohort study examining mobilisation practises and barriers. Crit Care. 2015;19(1):336. https://doi. org/10.1186/s13054-015-1033-3

8. Aquim EE, Bernardo WM, Buzzini RF, Azeredo NSG, Cunha LS, Damasceno MCP, et al. Brazilian guidelines for early mobilization in intensive care unit. Rev Bras Ter Intensiva. 2019;31(4):434-43. https://doi.org/10.5935/0103-507X.20190084
9. Alves GA, Martinez BP, Lunardi AC. Assessment of the measurement properties of the Brazilian versions of the Functional Status Score for the ICU and the Functional Independence Measure in critically ill patients in the intensive care unit. Rev Bras Ter Intensiva. 2019;31(4):521-8. https://doi. org/10.5935/0103-507X.20190065

10. Parry SM, Granger CL, Berney S, Jones J, Beach L, El-Ansary $D$, et al. Assessment of impairment and activity limitations in the critically ill: a systematic review of measurement instruments and their clinimetric properties. Intensive Care Med. 2015;41:744-62. https://doi.org/10.1007/s00134-015-3672-x

11. Dafoe S, Chapman MJ, Edwards S, Stiller K. Overcoming barriers to the mobilisation of patients in an intensive care unit. Anaesth Intensive Care. 2015;43(6):719-27. https://doi. org/10.1177/0310057x1504300609

12. Fontela PC, Forgiarini Júnior LA, Friedman G. Clinical attitudes and perceived barriers to early mobilization of critically ill patients in adult intensive care units. Rev Bras Ter Intensiva. 2018;30(2):187-94. https://doi.org/10.5935/0103-507X.20180037

13. van Aswegen $\mathrm{H}$, Patman S, Plani N, Hanekom S. Developing minimum clinical standards for physiotherapy in South African ICUs: A qualitative study. J Eval Clin Pract. 2017;23(6):1258-65. https://doi.org/10.1111/jep.12774

14. Nozawa E, Sarmento GJV, Vega JM, Costa D, Silva JEP, Feltrim MIZ. Perfil de fisioterapeutas brasileiros que atuam em unidades de terapia intensiva. Fisioter Pesqui. 2008;15(2):177-82. https:// doi.org/10.1590/s1809-29502008000200011

15. Lima AMS, Brandão DC, Barros CESR, Richtrmoc MKF, Andrade AFD, Campos SL. Knowledge of physiotherapists working in adult ICU on contraindications to mobilization. Fisioter mov. 2020;33:e003373. https://doi.org/10.1590/1980-5918.033.ao72

16. França EÉT, Ferrari F, Fernandes $P$, Cavalcanti R, Duarte A, Martinez BP, et al. Fisioterapia em pacientes críticos adultos: recomendações do Departamento de Fisioterapia da Associação de Medicina Intensiva Brasileira. Rev bras ter intensiva. 2012;24(1):6-22. http://dx.doi.org/10.1590/S0103507X2012000100003

17. Koo KKY, Choong K, Cook DJ, Herridge M, Newman A, Lo $\checkmark$, et al. Early mobilization of critically ill adults: a survey of knowledge, perceptions and practices of Canadian physicians and physiotherapists. C Open. 2016;4(3):E448-54. https://doi. org/10.9778/cmajo.20160021

18. Drolet A, DeJuilio P, Harkless S, Henricks S, Kamin E, Leddy EA, et al. Move to improve: The feasibility of using an early mobility protocol to increase ambulation in the intensive and intermediate care settings. Phys Ther 2013;93(2):197-207. https:// doi.org/10.2522/ptj.20110400 
19. Roque SM. Utilização do Escore Medical Research council (MRC ) e da Dinamometria de Preensão palmar no diagnóstico de fraqueza muscular adquirida em unidade de terapia intensiva (UTI): Revisão Bibliográfica [monografia] [Internet]. São Paulo: Faculdade Ávila; 2017. Disponível em: https://portalbiocursos. com.br/ohs/data/docs/236/25-UtilizaYYo_do_Escore_Medical_ Research council MRC e da dianamometria de preensYo palmar_no_diagnostico_de_fraqueza_muscular_adquirida_em_ unidade_de_terapia_intensivauti_revisYo_bibliografica.pdf

20. Gosselink R, Bott J, Johnson M, Dean E, Nava S, Norrenberg $M$, et al. Physiotherapy for adult patients with critical illness: Recommendations of the European Respiratory Society and European Society of Intensive Care Medicine Task Force on Physiotherapy for Critically III Patients. Intensive Care Med. 2008;34(7):1188-99. https://doi.org/10.1007/s00134-008-1026-7

21. Hickmann CE, Montecinos-Munoz NR, Castanares-Zapatero D, Arriagada-Garrido RS, Jeria-Blanco U, Gizzatullin T, et al. Acute Effects of Sitting Out of Bed and Exercise on Lung Aeration and Oxygenation in Critically III Subjects. Respir Care. 2020;66(2):25362. https://doi.org/10.4187/respcare.07487

22. Rocha ARM, Martinez BP, Silva VZM, Forgiarini Junior LA. Early mobilization: Why, what for and how? Med Intensiva. 2017;41(7):429-36. https://doi.org/10.1016/j.medin.2016.10.003

23. Pires-Neto RC, Kawaguchi YMF, Hirota AS, Fu C, Tanaka C, Caruso P, et al. Very Early Passive Cycling Exercise in Mechanically Ventilated Critically III Patients: Physiological and Safety Aspects - A Case Series. PLoS One. 2013;8:1-7. https://doi.org/10.1371/ journal.pone.0074182
24. Hodgson CL, Stiller K, Needham DM, Tipping CJ, Harrold M, Baldwin $\mathrm{CE}$, et al. Expert consensus and recommendations on safety criteria for active mobilization of mechanically ventilated critically ill adults. Crit Care. 2014;18(658):1-9. https://doi. org/10.1186/s13054-014-0658-y

25. Witcher R, Stoerger L, Dzierba AL, Silverstein A, Rosengart A, Brodie $D$, et al. Effect of early mobilization on sedation practices in the neurosciences intensive care unit: A preimplementation and postimplementation evaluation. J Crit Care. 2015;30(2):344-7. https://doi.org/10.1016/j.jcrc.2014.12.003

26. Dubb R, Nydahl P, Hermes C, Schwabbauer N, Toonstra A, Parker AM, et al. Barriers and strategies for early mobilization of patients in intensive care units. Ann Am Thorac Soc. 2016;13:72430. https://doi.org/10.1513/AnnalsATS.201509-586CME

27. Nassar Junior AP, Park M. Sedation protocols versus daily sedation interruption: A systematic review and metaanalysis. Rev Bras Ter Intensiva. 2016;28(4):444-51. https://doi. org/10.5935/0103-507X.20160078

28. Gonçalves DC, Martins DLB. Mobilização precoce no doente crítico: atualização. Scire Salut. 2018;8(1):16-24. https://doi. org/10.6008/cbpc2236-9600.2018.001.0003 